\title{
A Computationally Efficient Meshless Local Petrov-Galerkin Method for Axisymmetric Problems
}

\author{
I.S. Raju ${ }^{*}$ and T. Chen ${ }^{\dagger}$ \\ NASA Langley Research Center \\ Hampton, VA 23681
}

\begin{abstract}
$\underline{\text { ABSTRACT }}$
The Meshless Local Petrov-Galerkin (MLPG) method is one of the recently developed element-free methods. The method is convenient and can produce accurate results with continuous secondary variables, but is more computationally expensive than the finite element method. To overcome this disadvantage, a simple Heaviside test function is chosen. The computational effort is significantly reduced by eliminating the domain integral for the axisymmetric potential problems and by simplifying the domain integral for the axisymmetric elasticity problems. The method is evaluated through several patch tests for axisymmetric problems and example problems for which the exact solutions are available. The present method yielded very accurate solutions. The sensitivity of several parameters of the method is also studied.
\end{abstract}

\section{INTRODUCTION}

The Meshless Local Petrov-Galerkin (MLPG) method is a promising numerical method to analyze potential and elasticity problems and is shown to yield accurate results [1-6]. In this method, a set of arbitrarily distributed nodes is used to interpolate the field variables. The method does not use either 'elements' or a back-ground mesh for integration and hence is truly meshless. The method, however, appears to be more computationally expensive than the finite element method. One of the reasons for the high computational cost is that accurate numerical integration is required to integrate the weak form of the governing equations. Thus, if the domain integrations are eliminated or simplified, the method can be made efficient. A choice of a "Heaviside" test function $[5,6]$ leads to the elimination of the domain integral in the weak form of the axisymmetric potential problems and substantially simplifies the integral for axisymmetric elasticity problems. The purpose of this paper is to present such a method that utilizes the Heaviside test function and evaluate its effectiveness for axisymmetric problems.

The outline of the paper is as follows. First, a brief overview of MLPG method is presented for axisymmetric potential and elasticity problems. The MLPG formulation with Heaviside function is presented, and various issues related to the use of a Heaviside test function are studied. Finally the effectiveness of the method is demonstrated using several numerical problems.

\section{OVERVIEW OF MLPG METHOD}

In this section, several basic concepts that are used in the MLPG method are briefly reviewed. The development of the weak forms of the solution for potential and elasticity problems is first presented. Next, the choice of the trial function for the primary variables using the moving least squares method is reviewed. In the classical element-free Galerkin methods, the test functions are chosen from the same space as the trial functions. In the current MLPG method, the test functions are chosen to be different from the trial functions, and the choice of the test functions is discussed next.

\section{Potential Problem}

Consider Poisson's equation for an axisymmetric problem bounded by a toroidal domain with its cross section defined by $\Omega$ as shown in Figure 1,

$$
\nabla^{2} u=g \text { in } \Omega
$$

with boundary conditions

$$
u=\bar{u} \quad \text { on } \Gamma_{\mathrm{u}} \text { and } q=\bar{q} \quad \text { on } \Gamma_{\mathrm{q}}
$$

where $\Gamma=\Gamma_{\mathrm{u}}+\Gamma_{\mathrm{q}}$ and $q=d u / d n$. The Laplacian in the cylindrical coordinate system is

\footnotetext{
* Senior Technologist, Structures and Materials Competency, Fellow AIAA.

$\uparrow$ Army Research Laboratory, MS-240, Analytical and Computational Methods Branch, NASA Langley Research Center.
} 
$\nabla^{2}=\frac{\partial^{2}}{\partial r^{2}}+\frac{1}{r} \frac{\partial}{\partial r}+\frac{1}{r^{2}} \frac{\partial^{2}}{\partial \theta^{2}}+\frac{\partial^{2}}{\partial z^{2}}$

and for the axisymmetric problem, Eq. (3) reduces to

$$
\nabla^{2}=\frac{\partial^{2}}{\partial r^{2}}+\frac{1}{r} \frac{\partial}{\partial r}+\frac{\partial^{2}}{\partial z^{2}}
$$

The solution for Eq. (1) is sought in a weighted residual manner as

$$
2 \pi \int_{\Omega}\left(\nabla^{2} u-g\right) \cdot v \cdot r \cdot d r \cdot d z=0
$$

where $u$ is the approximate solution of the boundaryvalue problems and $v$ is a weight function.

Using the divergence theorem, one can rewrite Eq. (5) (dropping the constant $2 \pi$ ) as

$$
\begin{gathered}
-\int_{\Omega}\left[\frac{\partial u}{\partial r} \frac{\partial v}{\partial r}+\frac{\partial u}{\partial z} \frac{\partial v}{\partial z}\right] \cdot r \cdot d r \cdot d z-\int_{\Omega} g \cdot v \cdot r \cdot d r \cdot d z \\
+\int_{\Gamma}\left[\frac{\partial u}{\partial r} n_{r}+\frac{\partial u}{\partial z} n_{z}\right] \cdot r \cdot v \cdot d \Gamma=0
\end{gathered}
$$

where $n_{r}$ and $n_{z}$ are the direction cosines of the normal to the domain boundary along the $r$ - and $z$-directions, respectively. Eq. (6) is the weak form of the governing equation for potential problems.

In the literature, the interpolations for the primary variables $u$ are customarily termed as trial functions and the choice of the weight functions $v$ as the test functions. In the MLPG method, these choices are made from different spaces. The test functions are chosen such that they are non-zero over an arbitrary shape of domain $\Omega_{\mathrm{s}}$ that is known as compact support domain. In general, the domain is chosen to be a welldefined geometric shape such as a circle, a square, a rectangle or an ellipse. (In this paper, a compact support in the shape of circle is used.) As such, the integrals over $\Omega$ in Eq. (6) reduce to integrals over $\Omega_{\mathrm{s}}$. Eq. (6) can be written as

$$
\begin{aligned}
& -\int_{\Omega_{s}}\left[\frac{\partial u}{\partial r} \frac{\partial v}{\partial r}+\frac{\partial u}{\partial z} \frac{\partial v}{\partial z}\right] \cdot r \cdot d r \cdot d z+ \\
& \quad \int_{\Gamma_{s}}\left[\frac{\partial u}{\partial r} n_{r}+\frac{\partial u}{\partial z} n_{z}\right] \cdot r \cdot v \cdot d \Gamma+ \\
& \int_{\Gamma_{s q}} \bar{q} \cdot r \cdot v \cdot d \Gamma-\int_{\Omega_{s}} g \cdot v \cdot r \cdot d r \cdot d z=0
\end{aligned}
$$

where $\Gamma_{s}$ is the boundary of the compact support domain $\Omega_{\mathrm{s}}$ and $\Gamma_{s q}$ is $\Gamma_{q} \cap \Gamma_{s}$ (see Fig. 1) and $\bar{q}$ is the prescribed flux.

\section{Elasticity Problem}

Consider a problem of axisymmetric elasticity in a toroidal body with its cross section defined by domain $\Omega$ (Fig. 1), bounded by $\Gamma$. The equations of equilibrium are

$\frac{\partial \sigma_{r}}{\partial r}+\frac{\partial \tau_{r z}}{\partial z}+\frac{1}{r}\left(\sigma_{r}-\sigma_{\theta}\right)+f_{r}=0$

$\frac{\partial \tau_{r z}}{\partial r}+\frac{\partial \sigma_{z}}{\partial z}+\frac{1}{r} \tau_{r z}+f_{z}=0$

in $\Omega$ where $\sigma_{r}, \sigma_{\theta}, \sigma_{z}$ and $\tau_{r z}$ are the stresses in cylindrical coordinates $(r, \theta, z)$ and $f_{r}, f_{z}$ are the body forces in radial $(r)$ and $\operatorname{vertical}(z)$ directions, respectively. The boundary conditions on $\Gamma$ can be written as

$$
\begin{gathered}
u_{i}=\bar{u}_{i} \quad \text { on } \Gamma_{\mathrm{u}} \\
\sigma_{i j} \cdot n_{j}=\bar{t}_{i} \quad \text { on } \Gamma_{\mathrm{q}}
\end{gathered}
$$

where $n_{j}$ are the direction cosines to the normal of the boundary, and $\bar{u}$ and $\bar{t}$ are prescribed displacements and tractions, respectively. The weighted residual form for axisymmetric elasticity problems can be obtained using

$2 \pi \int_{\Omega}\left(\frac{\partial \sigma_{r}}{\partial r}+\frac{\partial \tau_{r z}}{\partial z}+\frac{1}{r}\left(\sigma_{r}-\sigma_{\theta}\right)+f_{r}\right) \cdot v_{r} \cdot r \cdot d r \cdot d z=0$

$2 \pi \int_{\Omega}\left(\frac{\partial \tau_{r z}}{\partial r}+\frac{\partial \sigma_{z}}{\partial z}+\frac{1}{r} \tau_{r z}+f_{z}\right) \cdot v_{z} \cdot r \cdot d r \cdot d z=0$

where $v_{r}$ and $v_{z}$ are weight functions for $r$-and $z$ directions, respectively. Using the divergence theorem, Eqs. (11) and (12) can be rewritten to obtain the weak form of the Eq.(8) as

$$
\begin{aligned}
& -\int_{\Omega}\left(\sigma_{r} \cdot \frac{\partial v_{r}}{\partial r}+\tau_{r z} \cdot \frac{\partial v_{r}}{\partial z}+\frac{\sigma_{\theta}}{r} \cdot v_{r}\right) \cdot r \cdot d r \cdot d z+ \\
& \int_{\Omega} f_{r} \cdot v_{r} \cdot r \cdot d r \cdot d z+ \\
& \quad \int_{\Gamma}\left(\sigma_{r} n_{r}+\tau_{r z} n_{z}\right) \cdot v_{r} \cdot r \cdot d \Gamma=0
\end{aligned}
$$




$$
\begin{gathered}
-\int_{\Omega}\left(\tau_{r z} \cdot \frac{\partial v_{z}}{\partial r}+\sigma_{z} \frac{\partial v_{z}}{\partial z}\right) \cdot r \cdot d r \cdot d z+ \\
\int_{\Omega} f_{z} \cdot v_{z} \cdot r \cdot d r \cdot d z+ \\
\int_{\Gamma}\left(\tau_{r z} n_{r}+\sigma_{z} n_{z}\right) \cdot v_{z} \cdot r \cdot d \Gamma=0
\end{gathered}
$$

By choosing the test functions $v_{r}$ and $v_{z}$ to be nonzero only inside a compact support domain $\Omega_{\mathrm{s}}$, the weak form (Eqs. (13) and (14)) can be rewritten as

$$
\begin{aligned}
- & \int_{\Omega_{s}}\left(\sigma_{r} \cdot \frac{\partial v_{r}}{\partial r}+\tau_{r z} \cdot \frac{\partial v_{r}}{\partial z}+\frac{\sigma_{\theta}}{r} \cdot v_{r}\right) \cdot r \cdot d r \cdot d z+ \\
& \int_{\Gamma_{s}}\left(\sigma_{r} n_{r}+\tau_{r z} n_{z}\right) \cdot v_{r} \cdot r \cdot d \Gamma+ \\
& \int_{\Omega_{s}} f_{r} \cdot v_{r} \cdot r \cdot d r \cdot d z+\int_{\Gamma_{s q}} \bar{t}_{r} \cdot v_{r} \cdot r \cdot d \Gamma=0 \\
- & \int_{\Omega_{s}}\left(\tau_{r z} \cdot \frac{\partial v_{z}}{\partial r}+\sigma_{z} \frac{\partial v_{z}}{\partial z}\right) \cdot r \cdot d r \cdot d z+ \\
& \int_{\Gamma_{s}}\left(\tau_{r z} n_{r}+\sigma_{z} n_{z}\right) \cdot v_{z} \cdot r \cdot d \Gamma+ \\
& \int_{\Omega_{s}} f_{z} \cdot v_{z} \cdot r \cdot d r \cdot d z+\int_{\Gamma_{s q}} \overline{t_{z}} \cdot v_{z} \cdot r \cdot d \Gamma=0
\end{aligned}
$$

where $\bar{t}$ are prescribed tractions on $\Gamma_{s q}$.

\section{Trial functions using Moving Least Squares} $\underline{\text { Interpolation }}$

The moving least squares (MLS) approximations have reasonably high accuracy and can be extended to $n$-dimensional problems, and hence are widely used to construct the trial functions in meshless methods [16]. This interpolation is used to represent the trial functions of the primary variables at a set of randomly located nodes in the domain. The interpolation function $u^{h}(\mathbf{x})$ of MLS method is defined as

$u^{h}(\mathbf{x})=\mathbf{p}^{T}(\mathbf{x}) \cdot \mathbf{a}(\mathbf{x}) \quad \forall \mathbf{x} \in \Omega$

where $\mathbf{p}^{\mathrm{T}}(\mathbf{x})$ is the monomial basis function of order $m$. For axisymmetric problems, monomial basis functions are chosen as

$$
\mathbf{p}^{T}(\mathbf{x})= \begin{cases}{[1, r, z]} & (m=3) \\ {\left[1, r, z, r^{2}, r z, z^{2}\right]} & (m=6) \\ {\left[1, r, z, r^{2}, r z, z^{2}, r^{3}, r^{2} z, r z^{2}, z^{3}\right]} & (m=10)\end{cases}
$$

where $m=3,6$, and 10 are complete linear, quadratic and cubic basis functions, respectively.
The coefficient vector $\mathbf{a}(\mathbf{x})$ in Eq. (17) can be obtained by minimizing the function $J(\mathbf{x})$ that is evaluated at $n$ discrete nodes

$J(\mathbf{x})=\sum_{i=1}^{n} w_{i}(\mathbf{x}) \cdot\left[\mathbf{p}^{T}\left(\mathbf{x}_{i}\right) \cdot \mathbf{a}(\mathbf{x})-\hat{\mathbf{u}}\right]^{2}$,

where $\hat{\mathbf{u}}$ is a vector of the fictitious nodal value of $u$, and $w_{i}(\mathbf{x})$ is a weight function. (Figure 2 illustrates the MLS scheme for a 1-D example.) In this paper, two spline functions, one with $\mathrm{C}^{1}$ and another with $\mathrm{C}^{2}$ continuity, are used as weight functions. The weight functions chosen are:

$C^{1}:$

$w_{k}(\mathbf{x})=\left\{\begin{array}{cr}1-3 \rho_{k}{ }^{2}+2 \rho_{k}^{3} & 0 \leq \rho_{k} \leq 1 \\ 0 & \rho_{k}>1\end{array}\right.$

$C^{2}$ :

$w_{k}(\mathbf{x})=\left\{\begin{array}{cc}1-10 \rho_{k}{ }^{3}+15 \rho_{k}{ }^{4}-6 \rho_{k}{ }^{5} & 0 \leq \rho_{k} \leq 1 \\ 0 & \rho_{k}>1\end{array}\right.$

where $\rho_{k}=\left(d_{k} / l_{k}\right)$ is the normalized distance, $d_{k}=\left\|\mathbf{x}-\mathbf{x}_{k}\right\|$ is the distance between center of the sub-domain of influence $\mathbf{x}_{k}\left(r_{k}, z_{k}\right)$ and a general point $\mathbf{x}$, and $l_{k}$ is the radius of the domain of influence (extent of the trial function) at node $k$ (see Figure 1).

By minimizing Eq.(19), then substituting the result into Eq.(17), the MLS interpolation function (see Fig. 2 for a one-dimensional example) is obtained as [1-6]

$u^{h}(\mathbf{x})=\sum_{i=1}^{n} \varphi_{i}(\mathbf{x}) \cdot \hat{u}_{i}$

where $n$ is the number of nodes in the domain, $\hat{u}_{i}$ is the fictitious nodal value of $u$ at node $i$, and $\varphi_{i}(\mathbf{x})$ is the shape function of node $i$, and is defined as

$$
\varphi_{i}(\mathbf{x})=\sum_{j=1}^{m} p_{j}(\mathbf{x}) \cdot\left[\mathbf{A}^{-1}(\mathbf{x}) \cdot \mathbf{B}(\mathbf{x})\right]_{j i}
$$

with

$$
\mathbf{A}(\mathbf{x})=\sum_{i=1}^{n} w_{i}(\mathbf{x}) \cdot \mathbf{p}^{T}\left(\mathbf{x}_{i}\right) \cdot \mathbf{p}\left(\mathbf{x}_{i}\right)
$$

$\mathbf{B}(\mathbf{x})=\left[w_{1}(\mathbf{x}) \cdot \mathbf{p}\left(\mathbf{x}_{1}\right), w_{2}(\mathbf{x}) \cdot \mathbf{p}\left(\mathbf{x}_{2}\right), \ldots w_{n}(\mathbf{x}) \cdot \mathbf{p}\left(\mathbf{x}_{n}\right)\right]$ 
Note that in the MLS approximation $u_{i}$ evaluated using Eq. (22) is not equal to the fictitious nodal vaule $\hat{u}_{i}$ (also see Figure 2). The partial derivatives of $\varphi_{i}(\mathbf{x})$ are needed in the weak form (Eqs.(7), (15) and (16)). Since both matrix $\mathbf{A}(\mathbf{x})$ and $\mathbf{B}(\mathbf{x})$ are functions of $\mathbf{x}$, the partial derivatives of $\varphi_{i}(\mathbf{x})$ have complicated form as [2]

$\varphi_{i, k}=\sum_{j=1}^{m}\left[p_{j, k}\left(\mathbf{A}^{-1} \mathbf{B}\right)_{j i}+p_{j}\left(\mathbf{A}^{-1} \mathbf{B}_{, k}+\mathbf{A}_{, k}^{-1} \mathbf{B}\right)_{j i}\right]$

where

$$
\mathbf{A}_{, k}^{-1}=\left(\mathbf{A}^{-1}\right)_{, k}=-\mathbf{A}^{-1} \mathbf{A}_{, k} \mathbf{A}^{-1}
$$

Note that $\varphi_{i, k}$ in Eq. (26) represent $\varphi_{i, r}$ and $\varphi_{i, z}$.

\section{Test function using Heaviside Function}

The next step in the MLPG method is to choose the test function. In the current implementation, a Heaviside function test function is chosen as the test function

$$
v(\mathbf{x})= \begin{cases}c & \mathbf{x} \in \Omega_{s} \\ 0 & \mathbf{x} \notin \Omega_{s}\end{cases}
$$

where $\mathrm{c}$ is an arbitrary constant ( $\mathrm{c}=1$ is used in this study). Using this choice, the partial derivatives of the test function $\partial v / \partial r$ and $\partial v / \partial z$ are identically zero, hence the domain integral involved in Eqs. (7) and (16) are identically zero, and Eq. (15) for elasticity problems is also considerably simplified. The elimination of domain integrals over $\Omega_{s}$ in Equations (7) and (16) considerably reduces the computing effort and hence makes this method computationally efficient.

For potential problems, Eq. (7) is simplified as

$$
\begin{gathered}
\int_{\Gamma_{s}}\left[\frac{\partial u}{\partial r} n_{r}+\frac{\partial u}{\partial z} n_{z}\right] \cdot r \cdot v \cdot d \Gamma+\int_{\Gamma_{s q}} \bar{q} \cdot r \cdot v \cdot d \Gamma- \\
\int_{\Omega_{s}} g \cdot v \cdot r \cdot d r \cdot d z=0
\end{gathered}
$$

and for elasticity problems, Equations (15) and (16) reduce to

$$
\begin{gathered}
-\int_{\Omega_{s}} \frac{\sigma_{\theta}}{r} \cdot v_{r} \cdot r \cdot d r \cdot d z+\int_{\Omega_{s}} f_{r} \cdot v_{r} \cdot r \cdot d r \cdot d z+ \\
\int_{\Gamma_{s}}\left(\sigma_{r} n_{r}+\tau_{r z} n_{z}\right) \cdot v_{r} \cdot r \cdot d \Gamma=0 \\
\int_{\Omega_{s}} f_{z} \cdot v_{z} \cdot r \cdot d r \cdot d z+ \\
\int_{\Gamma_{s}}\left(\tau_{r z} n_{r}+\sigma_{z} n_{z}\right) \cdot v_{z} \cdot r \cdot d \Gamma=0
\end{gathered}
$$

In this paper, the MLPG algorithm using the Heaviside test function is applied to various axisymmetric problems to study salient features of the method. The sensitivity of the MLPG solution to various parameters such as the size of the compact support domains, the basis function, etc., will be presented to demonstrate the algorithm.

\section{EXAMPLES}

To evaluate the current MLPG algorithm for potential and elasticity problems, several patch test problems with exact solutions are considered. The sensitivity of the MLPG solution to the basis functions and the two weight functions $w_{k}(\boldsymbol{R})$ are studied. Since all of the potential and elasticity problems in this study are linear problems, the dimensions of the patch test models are multiples of a constant $a$. Various normalized values for the radii of domain of the influence, $\left(l_{k} / a\right)$, and the radii of the support domains, $\left(l_{o} / a\right)$, are used to study their influence on the accuracy of the results.

The results of the MLPG method are compared to exact solutions. The error norm $\left(\left\|e_{M}\right\|_{2}\right)$ is used to evaluate the effects of various parameters, and is given by

$\left\|e_{M}\right\|_{2}=\sqrt{\sum_{j=1}^{M}\left\{\left(u_{j}-u_{\text {exact }}\right) / u_{\text {exact }}\right\}^{2} / M}$,

where $M$ is the total number of randomly distributed internal points in the domain at which the numerical solution is evaluated and compared to the exact solution, $u_{\text {exact }}$. Note that these internal points are independent points and are not associated with the nodes used in the MLPG models. A value of $\mathrm{M}=50$ is used for all models.

\section{Potential Problem}

\section{Patch tests}

Two patch tests involving Laplace- $\left(\nabla^{2} u=0\right)$, and Poisson- $\left(\nabla^{2} u=\right.$ constant $)$ equation problems are considered. Figure 3 shows two models used in the 
patch tests of the Laplace and Poisson equation problems. These examples are analyzed using the MLPG algorithm for various combinations of prescribed boundary conditions involving $u$ and $q$. The Laplace equation over a triangular domain with two mixed boundary conditions is studied using a quadratic basis function. An exact solution for this problem is $u=r^{2}-2 z^{2}+3 z$. The radius of the domain of influence $l_{k}$ is set to $4 a$. Two different choices for $l_{o}$ are considered. In the first choice, the distance from each internal node to the closest boundary is computed. These distances are used as the values of $l_{o}$ for that node. Using this definition, the support domain radius may be different for each node. The MLPG algorithm with different values of $l_{o}$ for each node recovered the exact solution for the Laplace equation problems. In the second choice, the smallest value of all the $l_{o}$ values from the first choice is used for all the nodes. Once again, the exact solution was recovered to machine accuracy. In these studies, the effect of the radius of the domain of influence, $l_{k}$, was also evaluated by varying the radius from $4 a$ to $10 a$. The accuracy of the results is insensitive to the radius of the domain of influence, $l_{k}$.

The sensitivity of the MLPG solution is further studied by varying the support domain radius $l_{o}$, and the error norm is shown in Figure 4. All of the nodes in the model were set to have the same value of $l_{0}$. The results show that, for the Laplace equation, the two weight functions considered with $\mathrm{C}^{1}$ and $\mathrm{C}^{2}$ continuity (Eqs.(20) and (21)) gave excellent solutions for both boundary value problems and for a variety of the normalized support domain radius, $l_{0} / a$.

The next problem considered involves a Poisson's equation, $\nabla^{2} u=8$, over the L-shaped domain shown in Fig. 3. An exact solution for this problem is $u=r^{2}+2 z^{2}+3 z-2$. The convergence of the solution for various values of $l_{o}$ and for different boundary conditions is presented in Figure 5. Once again, the exact solution is recovered by MLPG algorithm for all idealizations demonstrating that the present MLPG algorithm is very accurate.

\section{Heat Transfer Example}

Figure 6 shows a heat transfer problem involving radial heat flow in a hollow circular cylinder with prescribed constant temperatures $T_{1}$ and $T_{2}$ at the inner and outer surfaces, respectively. The exact solution for the temperature at radius $r$ is defined as

$$
T=T_{1}-\left(T_{1}-T_{2}\right) \cdot \frac{\ln \left(r / r_{1}\right)}{\ln \left(r_{2} / r_{1}\right)}
$$

where $r_{1}$ and $r_{2}$ are the inner and outer radii, respectively. The heat flux, $q$, is defined as

$q=-\frac{\left(T_{1}-T_{2}\right)}{r \cdot \ln \left(r_{2} / r_{1}\right)}$

The MLPG model with 25 randomly distributed nodes and the boundary conditions are also shown in Figure 6. The exact solution for the temperature is a natural logarithmic function, and since the natural logarithmic function cannot be exactly represented by a polynomial, a study of the use of various basis functions (polynomial functions) is undertaken. Three polynomial basis functions, linear, quadratic, and cubic, are considered (Eq. (18)). Note that the higher order basis function requires substantially more computational effort than the lower order basis function. Figure 7 shows the error norm for various basis functions with a $\mathrm{C}^{1}$ weight function. The support domain radius, $l_{0}$, is set equal to $0.35 a$ for the nodes on the boundary and equal to the distance to the closest boundary for the internal nodes. Accurate solutions are obtained for all cases studied. Figure 7 shows that the higher order basis functions yielded more accurate results than the lower order basis functions.

\section{Elasticity Problems}

For all the elasticity problems studied, a Young's modulus, $E=100 \mathrm{GPa}$, and a Poisson's ratio, $v=0.3$ are used.

\section{Patch Test}

The axisymmetric elastic MLPG method was evaluated for patch test problems with the displacements $\left(u_{r}=c_{1} \cdot r ; u_{z}=c_{2} \cdot z\right)$ of a circular cylinder with different combinations of boundary conditions. Since exact solutions for these problems can be represented by polynomial functions, the MLPG method recovered the solutions to machine accuracy for all weight and basis functions considered. As in the cases of the potential problems, the accuracy of the results is insensitive to the radius of the domain of influence, $l_{k}$.

\section{Lamé's Cylinder}

A hollow cylinder subjected to constant internal $\left(p_{1}\right)$ and external $\left(p_{2}\right)$ pressures (Lamé's Problem) is considered (see Figure 8). For the problem analyzed, the inner radius $r_{1}$ and the outer radius $r_{2}$ are $3 a$ and $6 a$, respectively, where $a$ is an arbitrary constant. The nodal arrangement is shown in Figure 8b. The exact solution of Lamé's problem is 


$$
\begin{aligned}
& \sigma_{r}=C_{1}-C_{2} / r^{2} ; \sigma_{\theta}=C_{1}+C_{2} / r^{2} ; \sigma_{z}=2 \cdot \nu \cdot C_{1} \\
& u_{r}=\frac{1+\nu}{E} \cdot\left[C_{1} \cdot(1-2 \nu) \cdot r+\frac{C_{2}}{r}\right] ; u_{z}=0 ;
\end{aligned}
$$

where

$$
C_{1}=\frac{p_{1} \cdot r_{1}^{2}-p_{2} \cdot r_{2}^{2}}{r_{2}^{2}-r_{1}^{2}} ; C_{2}=\frac{p_{1}-p_{2}}{r_{2}^{2}-r_{1}^{2}} \cdot r_{1}^{2} \cdot r_{2}^{2}
$$

Figure 9 shows the variation of the error norm for three cases using a linear basis function. In the first two cases, the trial functions are formed using $\mathrm{C}^{1}$ and $\mathrm{C}^{2}$ continuity weight functions. The Heaviside test function is used in both cases. In the third case, the linear basis function and $\mathrm{C}^{1}$ continuity weight function are used to form the trial function, and a $C^{1}$ spline test function is chosen as in reference [4]. All of the nodes in the model were chosen to have the same support domain radius, $l_{0}$. As seen in the figure, the error norm varies substantially as the support domain size changes for all of the cases. For the third case that utilizing the $\mathrm{C}^{1}$ spline test function, the error norm decreases monotonically as the normalized support domain radius increases. However, for the problems that used Heaviside test function, the error norm does not decrease monotonically. The error norm for the cases using the Heaviside test function reaches a minimum value near $l_{0} / a=0.6$, then begins increasing again as the support domain radius increases further. The accuracy decreases for values of $\left(l_{o} / a\right)>0.6$. This type of behavior was noted only for the results obtained using the Heaviside test function. Figure 9 also shows that the error norm of the solutions obtained using the $\mathrm{C}^{1}$-weight function is lower than that with the $\mathrm{C}^{2}$-weight function for linear basis function.

The effects of the basis function are also evaluated. The error norm obtained with each of the basis functions is presented in Figure 10. The exact solution for the displacement in Lamé's problem cannot be represented by any of the three - linear, quadratic and cubic - polynomial basis functions. Unlike for most problems studied, the linear basis function yielded the most accurate results, while the quadratic and cubic basis functions produced similar results.

\section{Rotating Disk}

The next problem studied is that of a hollow disk rotating about the $z$-axis with a radial velocity of $\omega$ $\mathrm{rad} / \mathrm{sec}$ as shown in Figure 11. In this problem, both top and bottom surfaces are constrained in the $z$ direction ("plane strain" condition) to simulate a uniformly rotating long cylinder. Both inner and outer surfaces are stress free, while the disk is subjected to an inertial force per unit volume that equals $\rho \cdot \omega^{2} \cdot r$, where $\rho$ is the density of the disk material and $\omega$ is the angular velocity. The exact solution of the problem is given by (see pages 335-337, Ref. [7])

$$
\begin{aligned}
& \sigma_{r}=\frac{(3-2 \cdot v)}{8 \cdot(1-v)} \cdot \rho \omega^{2}\left(r_{1}^{2}+r_{2}^{2}-\frac{r_{1}^{2} \cdot r_{2}^{2}}{r^{2}}-r^{2}\right) \\
& \sigma_{\theta}=\frac{(3-2 \cdot v)}{8 \cdot(1-v)} \cdot \rho \omega^{2}\left(r_{1}^{2}+r_{2}^{2}+\frac{r_{1}^{2} \cdot r_{2}^{2}}{r^{2}}-\frac{1+2 \cdot v}{(3-2 \cdot v)} r^{2}\right) \\
& \sigma_{z}=v \cdot\left(\sigma_{r}+\sigma_{\theta}\right) ; \quad u_{z}=0 \\
& u_{r}=r \cdot \varepsilon_{\theta}= \\
& \frac{(1+v) \cdot(3-2 v)}{8 E} \cdot \rho \omega^{2}\left[r \cdot\left(r_{1}^{2}+r_{2}^{2}\right)+\frac{r_{1}^{2} \cdot r_{2}^{2}}{r}-\frac{(1+2 v)}{(3-2 v)} r^{3}\right]- \\
& \frac{v \cdot(1+v) \cdot(3-2 v)}{8(1-v) E} \cdot \rho \omega^{2}\left[r \cdot\left(r_{1}^{2}+r_{2}^{2}\right)-\frac{r_{1}^{2} \cdot r_{2}^{2}}{r}-r^{3}\right]
\end{aligned}
$$

where $E$ is Young's modulus of the material and $v$ is Poisson's ratio.

The three models in Figure 11 are used to study the convergence of the solution to nodal refinement. In Model-1, seven nodal points are placed in the $r$ direction. In Model-2, the distance between adjacent nodes is half of the distance between adjacent nodes in Model-1 ( $\Delta / 2)$. In Model-3, the distance between adjacent nodes is a quarter of the distance in Model-1 $(\Delta / 4)$. The number of nodes in the $z$ direction is kept the same (equal to 5) for all of the models.

Figure 12 shows the effect of the nodal arrangement on the error norm. Model-3 has the most nodal points and produced, as expected, the most accurate solution. However, the analysis using Model-3 requires substantially more CPU time than that for Model-1. The effect of the basis functions is shown in Figure 13 using Model-2 with the $\mathrm{C}^{1}$ weight function. The cubic basis function yielded a more accurate result than either the linear or the quadratic basis function. However, as shown in Figure 13, the analysis using the linear basis function is only marginally less accurate than the cubic basis function and the linear basis requires considerably less computational effort than the analysis using the higher order basis function. Thus, the use of the linear basis function is preferred for this problem.

Figure 14 shows the effect of the support domain size on the error norm using Model-2 and a cubic 
basis function. The results, once again, show that the error norm pass through a minimum value and then begins to rise as the support domain radius increases past a value $l_{o} / a=0.18$.

\section{CONCLUDING REMARKS}

A meshless Local Petrov-Galerkin (MLPG) method that uses a Heaviside test function is presented and used to analyze potential and elasticity problems in axisymmetric domains. By using a Heaviside test function for the axisymmetric potential problems, the domain integral in the weak form is eliminated and a line integral is used instead. This substantially reduces the computation effort to construct the 'stiffness' matrix and hence is computationally efficient compared to the conventional MLPG method. In the axisymmetric elasticity problems, since the domain integral is simplified but is not eliminated, the reduction of the computational effort is limited. The MLPG algorithm using a Heaviside test function is implemented and its efficiency and accuracy are studied with respect to three basis functions, two different weight functions, and different sizes of the local support domain.

Potential patch test problems involving Laplace and Poisson equations are used to evaluate the efficiencies of the algorithm. To evaluate the effectiveness of the MLPG algorithm for elasticity problems, several simple patch test problems, a Lamé cylinder problem and a rotating disk problem, are studied.

The $\mathrm{C}^{1}$ weight function yielded more accurate results than the $\mathrm{C}^{2}$ functions for the patch test problems studied. Since the analysis using the $\mathrm{C}^{1}$ function requires less computing time than that using the $\mathrm{C}^{2}$ functions, the $\mathrm{C}^{1}$ function is recommended. Unlike the MLPG method that uses a local spline test function (Ref. [4]), the error norm for the MLPG method with the Heaviside test function passes through a minimum value as the support domain radius, $l_{o}$, increases. For the problems studied, the variation of the radius of the domain of influence, $l_{k}$, does not show a significant effect on the accuracy of the results. For the problems studied, the use of the cubic basis function did not always yield the most accurate results and substantially increases the computing time compared to the use of the linear basis function. In general, increasing the number of the nodes in the model increases the accuracy of the result.

In summary, the MLPG algorithm with a Heaviside test function yielded accurate solutions for the potential and elasticity patch tests as well as the other problems studied. A simple $\mathrm{C}^{1}$ weight function, and a simple linear basis function are recommended to obtain accurate results.

\section{REFERENCES}

[1] Nayroles, B., Touzot, G., and Villon, P., "Generalizing the Finite Element Method: Diffuse Approximation and Diffuse Elements," Computational Mechanics, Vol. 10, No. 5, 1992, pp. 307-318.

[2] Belytschko, T., Lu, Y.Y., and Gu, L., "ElementFree Galerkin Methods," International Journal for Numerical Methods in Engineering, Vol. 37, No. 2, 1994, pp. 229-256.

[3] Atluri, S.N., and Zhu, T., "A New Meshless Local Petrov-Galerkin (MLPG) Approach in Computational Mechanics," Computational Mechanics, Vol. 22, No. 2, 1998, pp. 117-127.

[4] Raju, I.S., and Chen, T., "Meshless PetrovGalerkin Method Applied to Axisymmetric Problems," Proceedings of the ICES 2K Conference, Los Angeles, CA, August 21-25, 2000.

[5] Atluri, S.N., and Shen, S., The Meshless Local Petrov-Galerkin (MLPG) Method, Tech. Science Press, Encino,CA, 2002.

[6] Atluri, S.N., and Shen, S., "The Meshless Local Petrov-Galerkin (MLPG) Methods: A Simple \& Less Costly Alternative to the Finite Element and Boundary Element Methods," Computer Modeling in Engineering \& Sciences, Vol. 3, No. 1, 2002, pp. 11-51.

[7] Saada, A.S., Elasticity: Theory and Applications, Robert E. Krieger Publishing Co., 1983. 


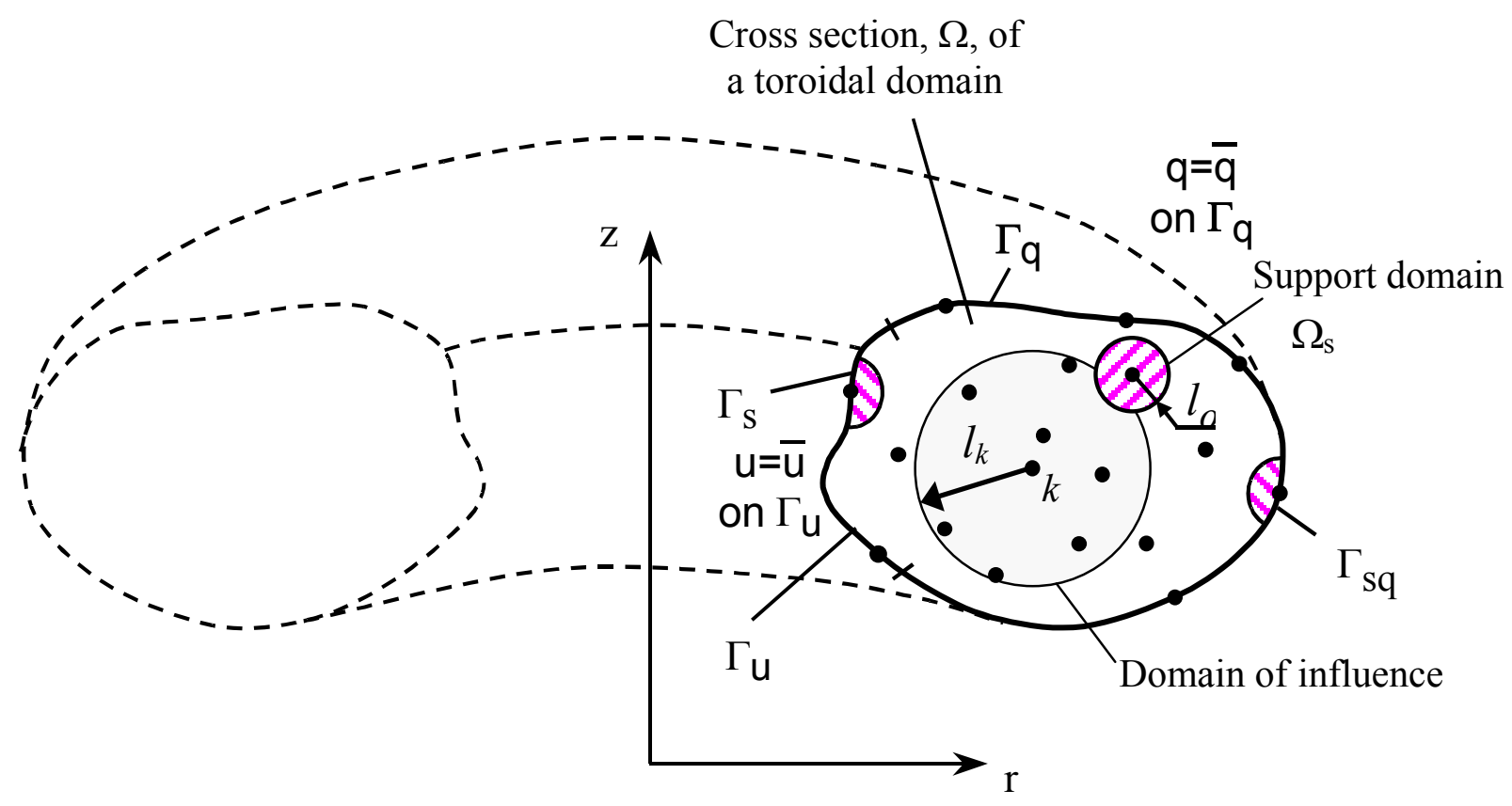

Figure 1 Typical nodal pattern for an axisymmetric potential problem

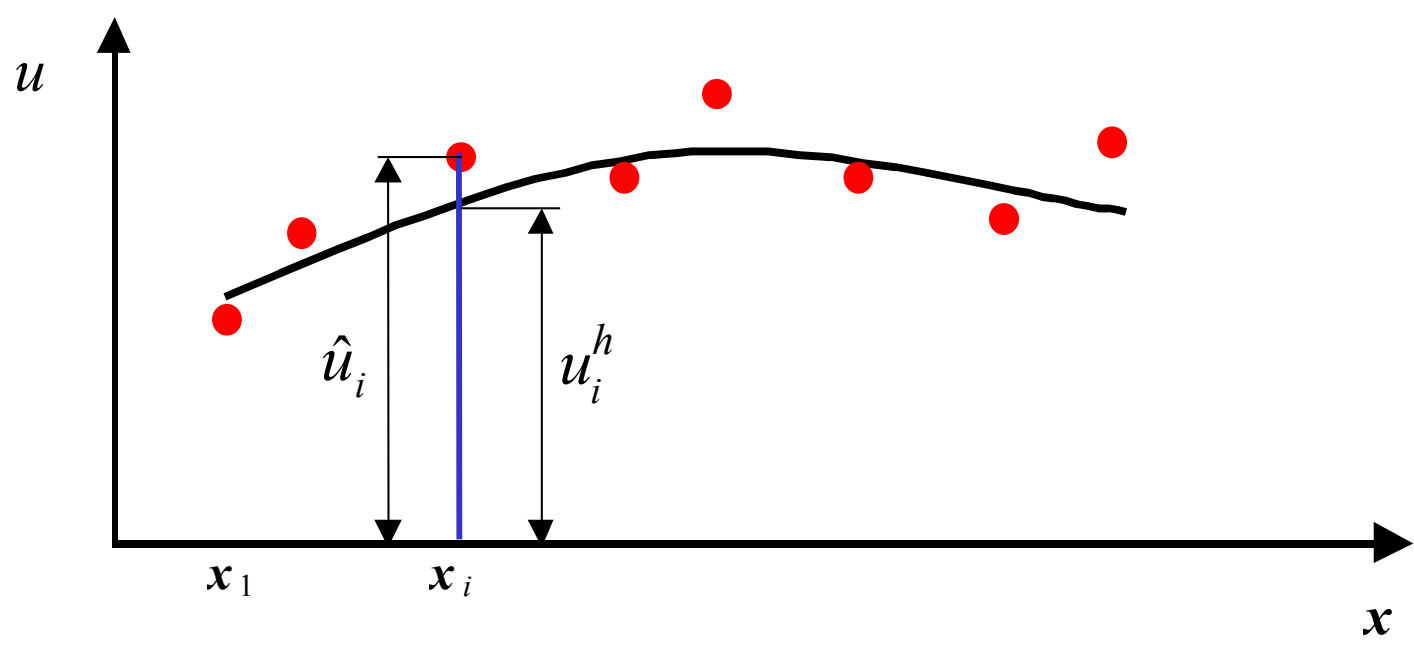

Figure 2 Approximation for $u$ using MLS scheme for a 1-D example 


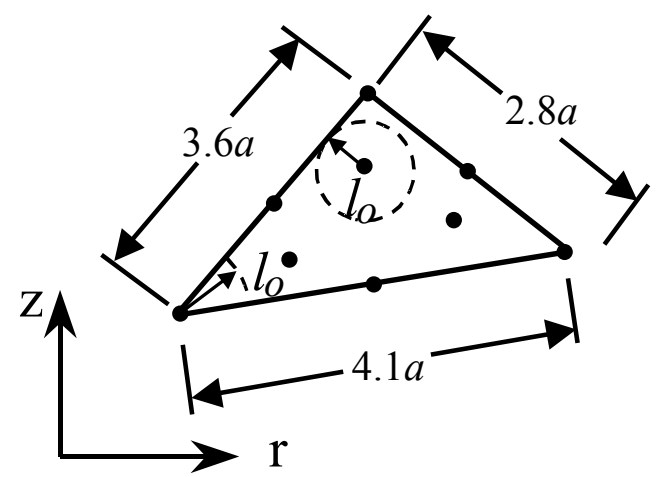

(a) Triangular domain

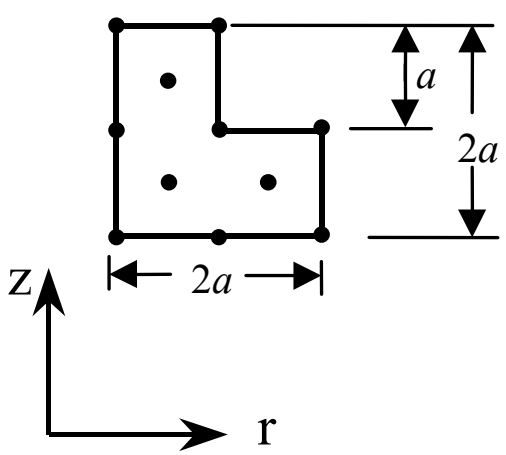

(b) L-shaped domain

Figure 3 Patch tests models for Laplace and Poisson's equations

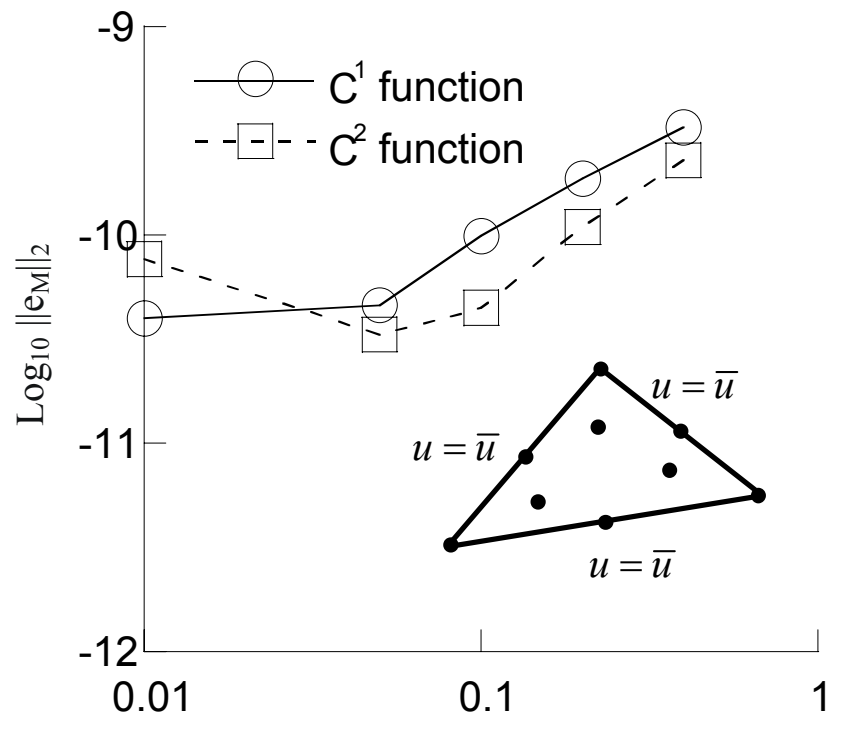

Normalized support domain, $l_{\delta} / a$

(a) Boundary value problem -1

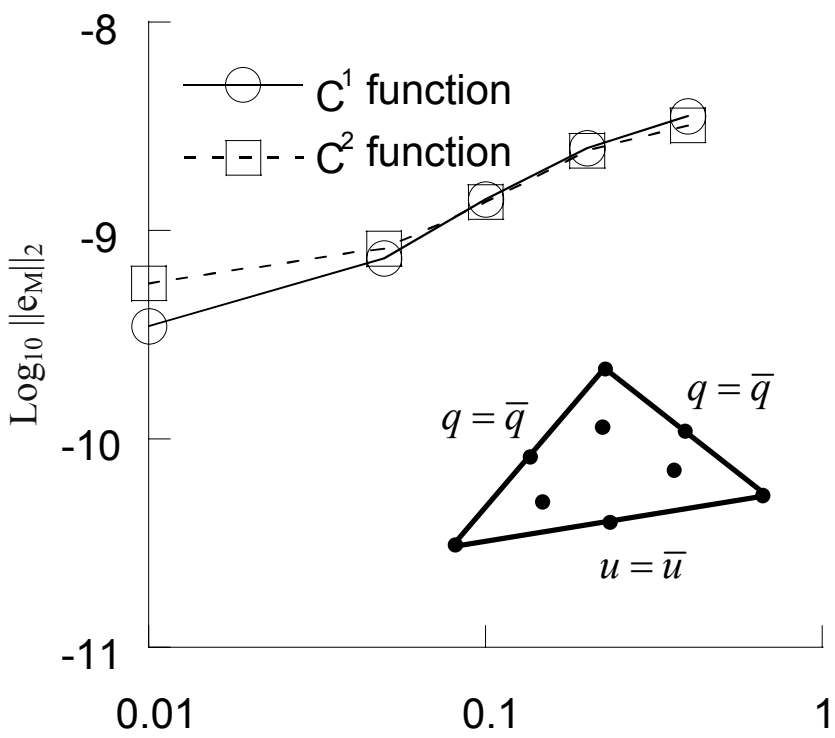

Normalized support domain, $l_{d} / a$

(b) Boundary value problem -2

Figure 4 Laplace problem with a triangular domain - Convergence of the solution for various values of $l_{o}\left(\nabla^{2} u=0\right.$; Quadratic basis; $\left.l_{k}=5 a\right)$ 


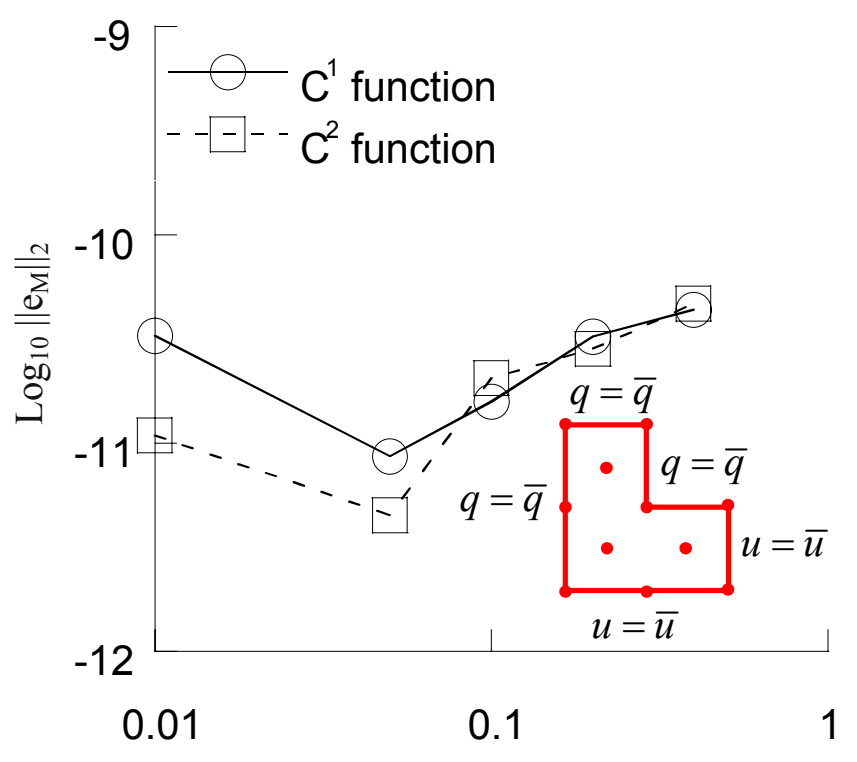

Normalized support domain, $l_{o} / a$

(a) Mixed boundary value problem -1

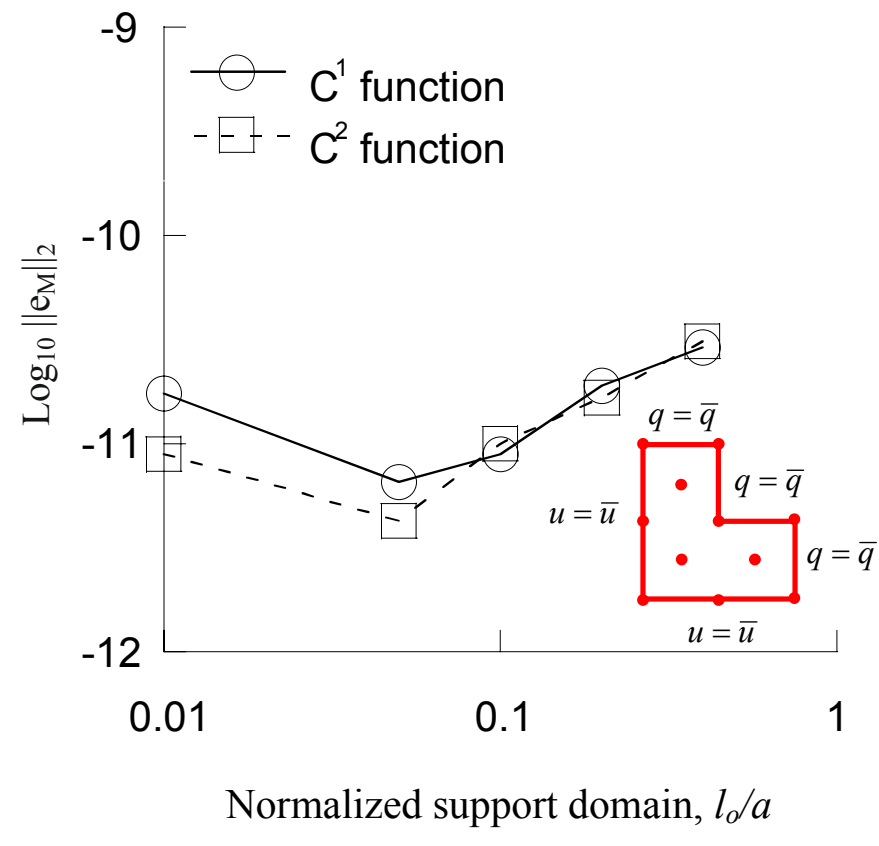

(b) Mixed boundary value problem -2

Figure 5 Poisson's problem with an L-shaped domain - Convergence of the solution for various values of $l_{o}\left(\nabla^{2} u=8\right.$; Quadratic basis; $\left.l_{k}=4 a\right)$

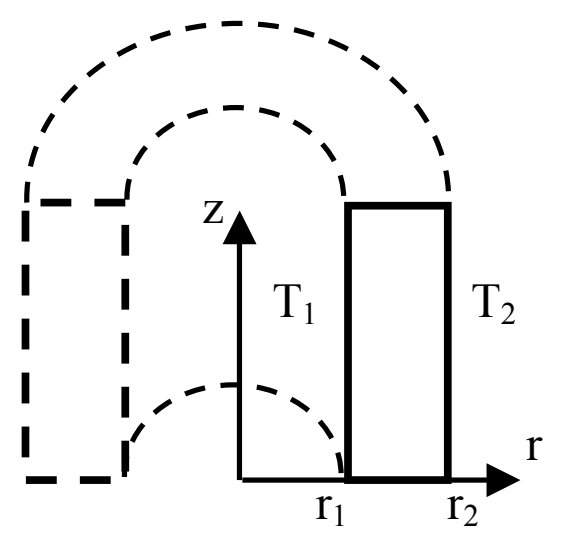

(a)

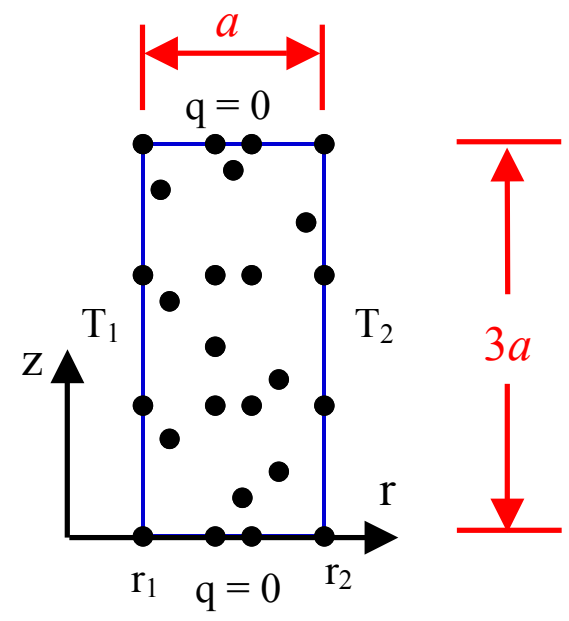

(b)

Figure 6 Heat transfer in a hollow circular cylinder 


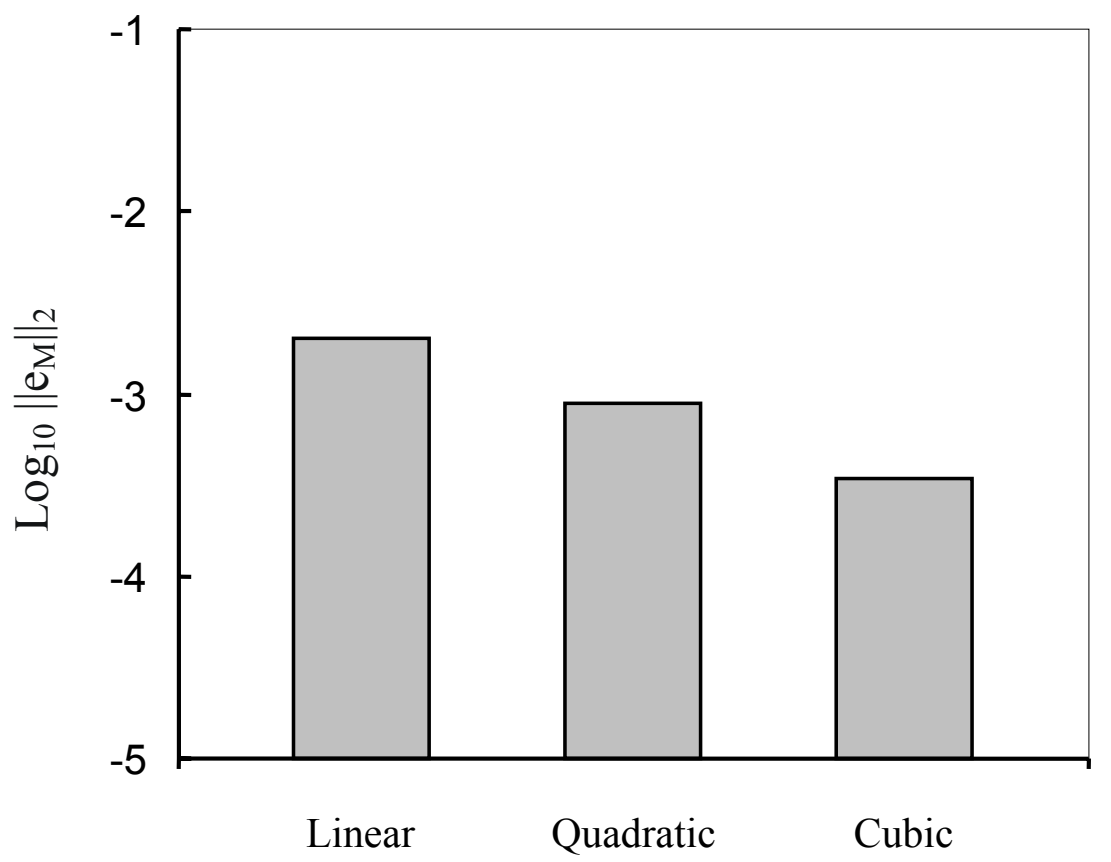

Figure 7 Heat transfer in a hollow circular cylinder Effect of basis functions ( $\mathrm{C}^{1}$ weight function)

(a) Làme's cylinder

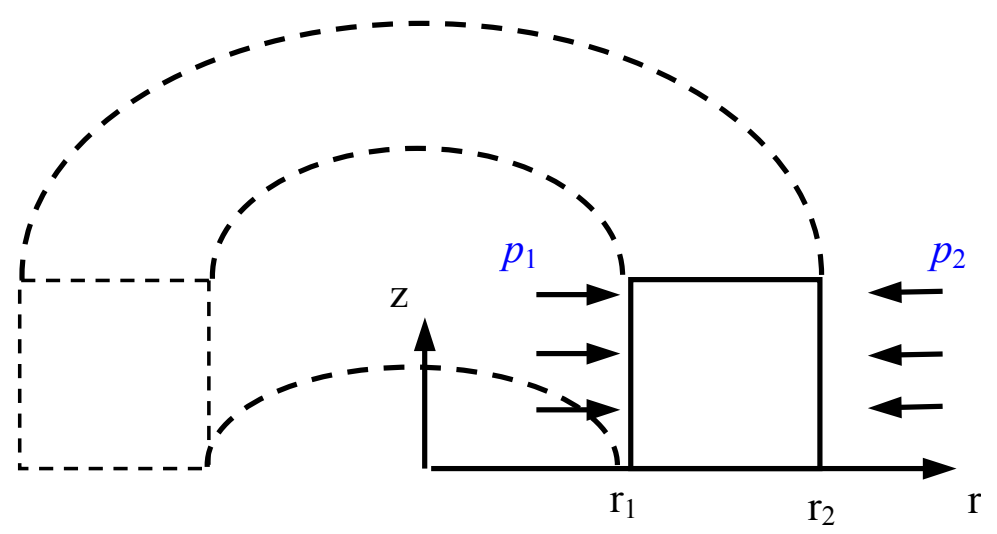

(b) Meshless model

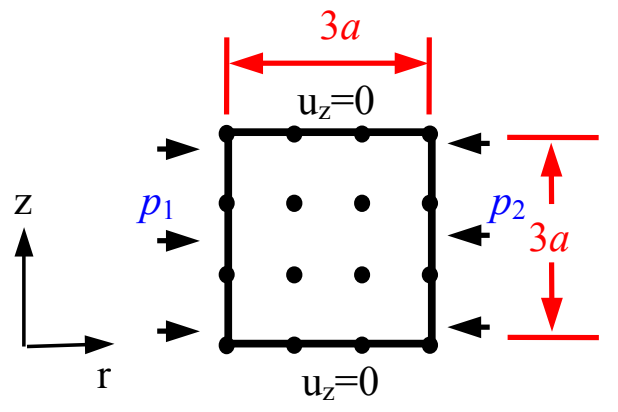

Figure 8 Hollow cylinder subjected to internal and external pressures (Làme's Problem) 

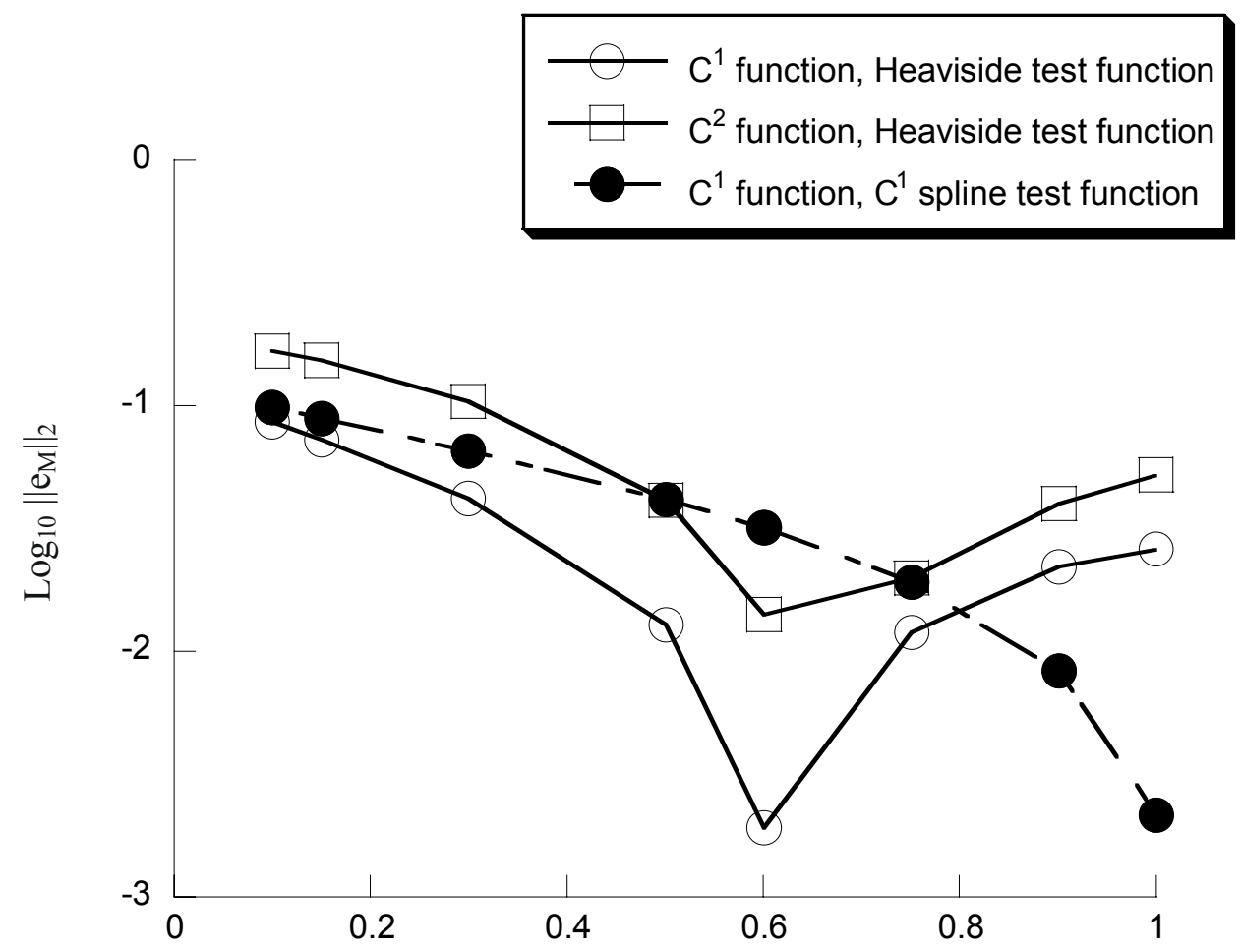

Normalized support domain radius, $l_{o} / a$

Figure 9 Lamé's cylinder - (linear basis; $\left.l_{k} / a=4\right)$

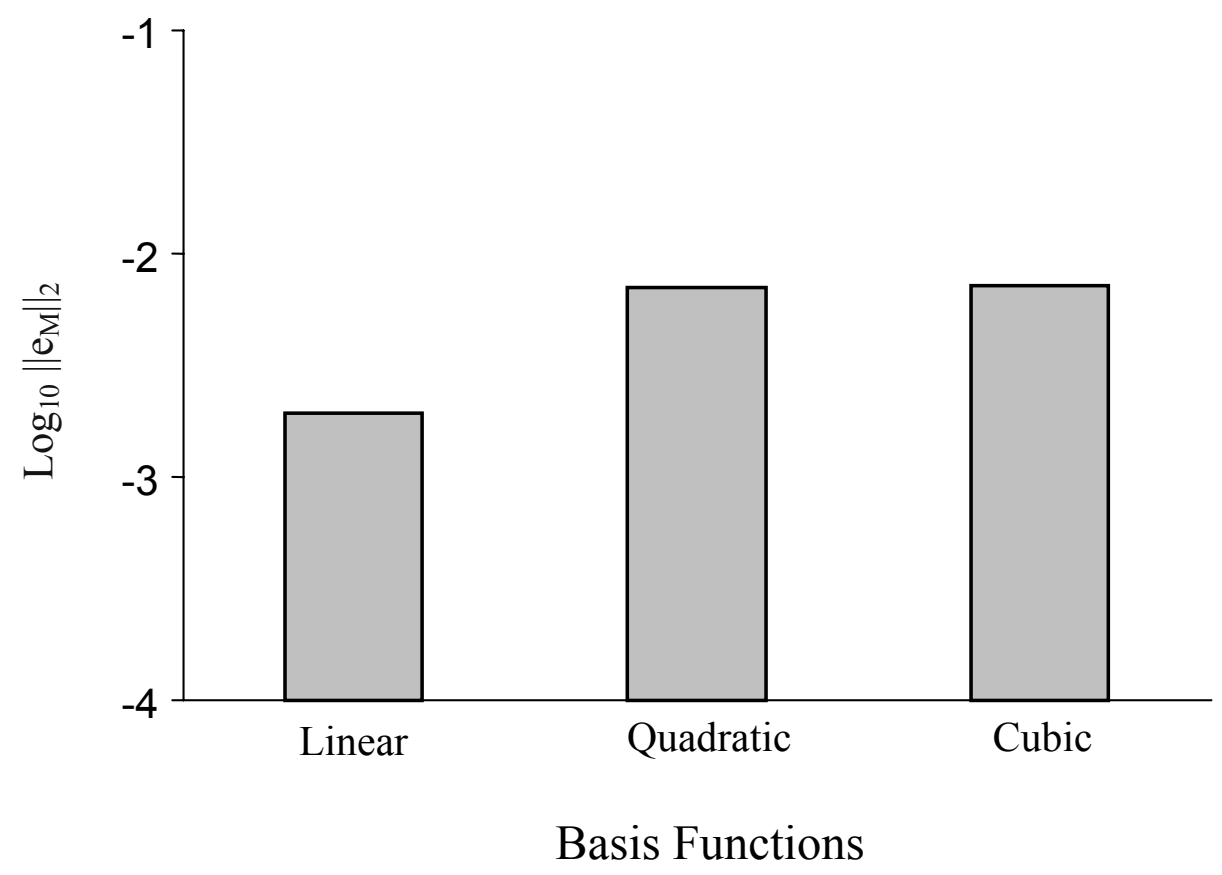

Figure 10 Làme's cylinder - Effects of basis functions $\left(\mathrm{C}^{1}\right.$ weight function; $l_{0} / a=0.6$ ) 

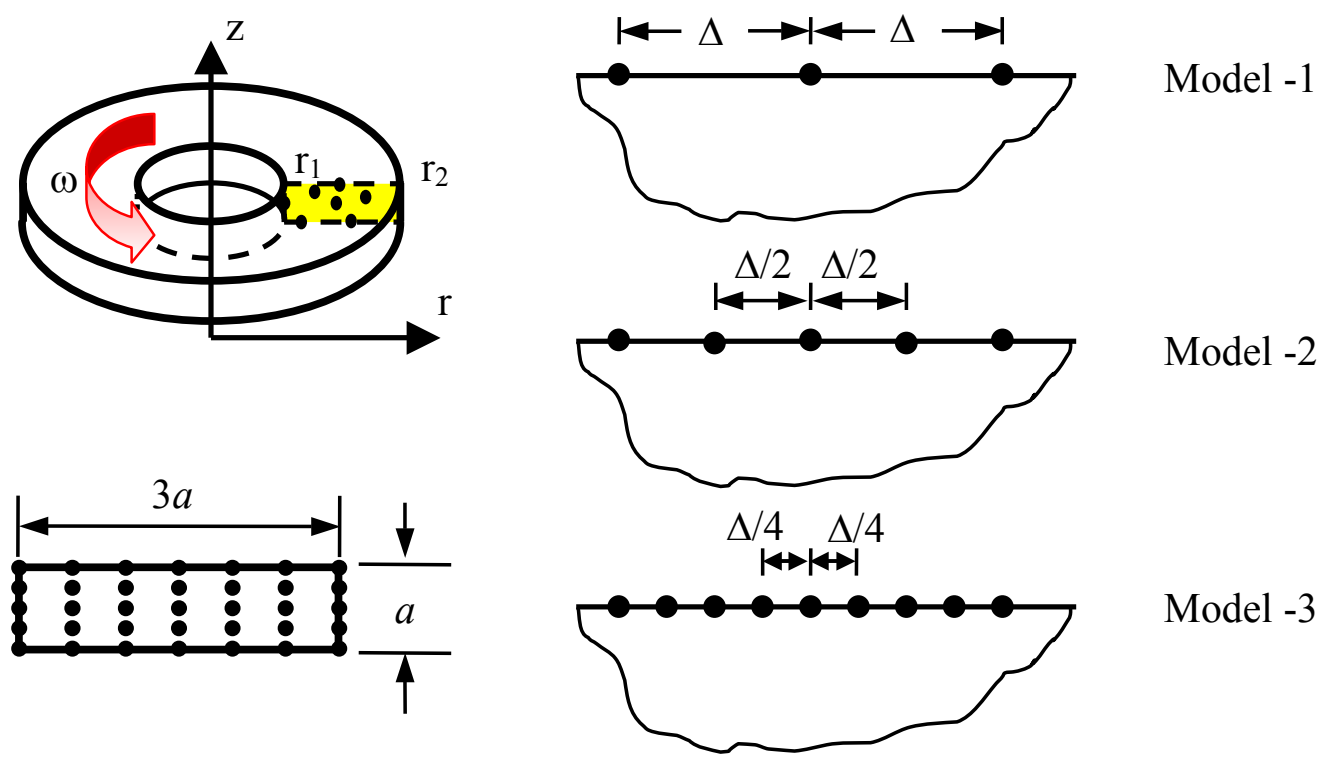

Figure 11 Rotating disk problem and the three models used

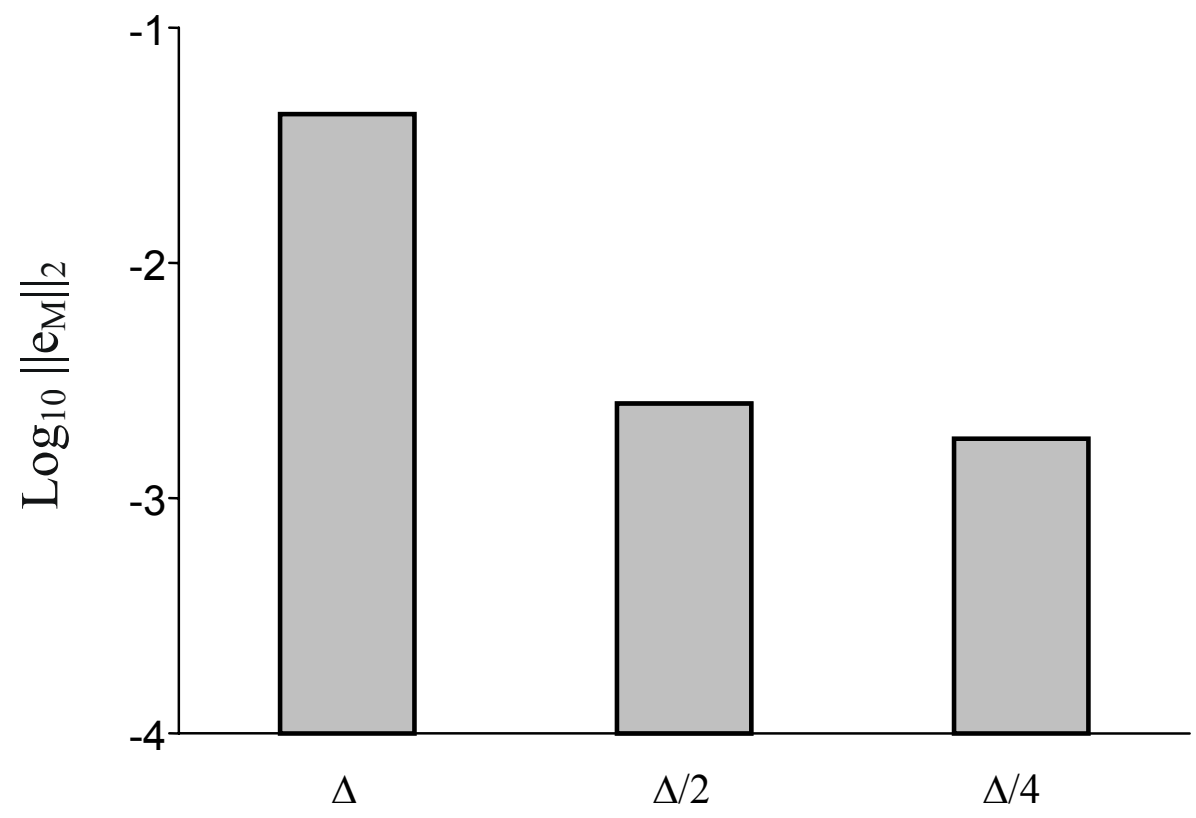

Distance between nodes in the $r$ - direction

Figure 12 Rotating disk - Effect of nodal refinement - $\left(C^{1}\right.$ weight function; Cubic basis function; $l_{o} / a=0.125$ ) 


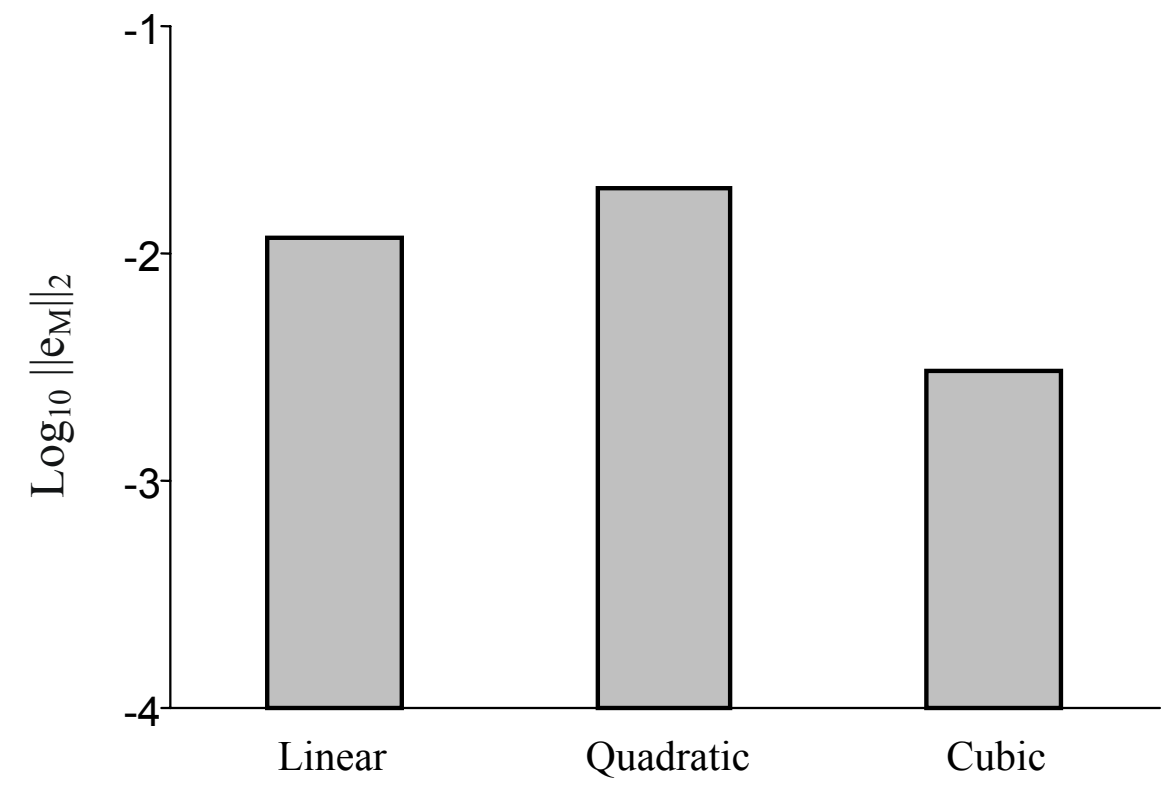

Basis functions

Figure 13 Rotating disk - Effect of basis functions - $\left(\mathrm{C}^{1}\right.$

weight function; $l_{o} / a=0.125$; Model-2)

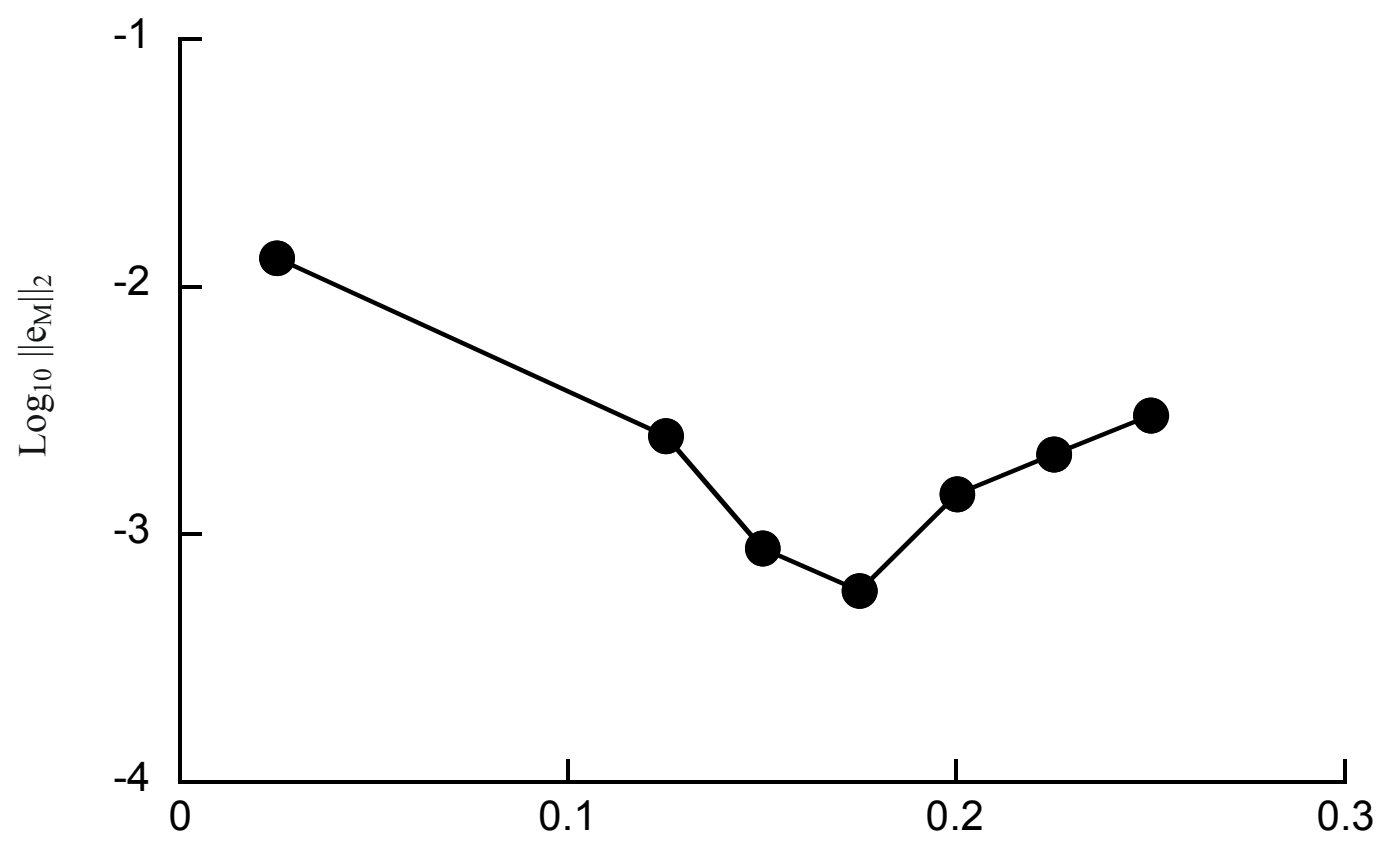

Support domain radius, $\left(l_{0} / a\right)$

Figure 14 Rotating disk - Effect of support domain radius - $\left(\mathrm{C}^{1}\right.$ weight function; Cubic basis function, Model-2) 